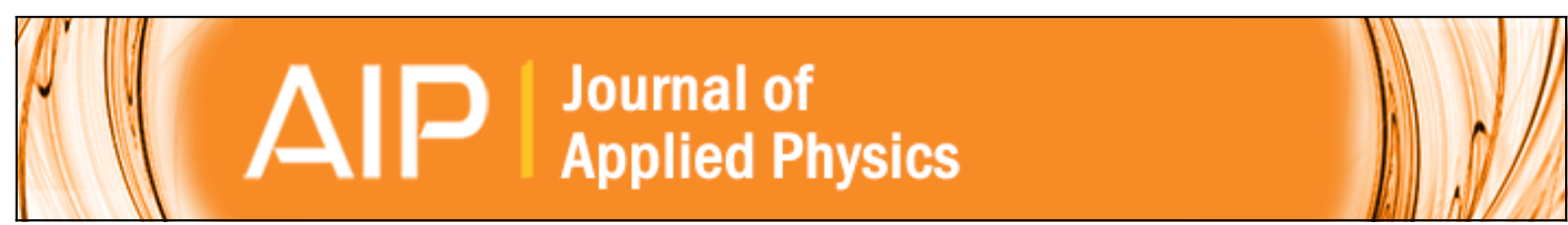

\title{
Observation of room temperature saturated ferroelectric polarization in Dy substituted
} BiFeO3 ceramics

Shuxia Zhang, Lei Wang, Yao Chen, Dongliang Wang, Yingbang Yao, and Yanwei Ma

Citation: Journal of Applied Physics 111, 074105 (2012); doi: 10.1063/1.3702405

View online: http://dx.doi.org/10.1063/1.3702405

View Table of Contents: http://scitation.aip.org/content/aip/journal/jap/111/7?ver=pdfcov

Published by the AIP Publishing

\section{Articles you may be interested in}

Significantly enhanced ferroelectricity and magnetic properties in ( $\mathrm{Sr} 0.5 \mathrm{Ca} 0.5) \mathrm{TiO} 3$-modified $\mathrm{BiFeO} 3$ ceramics J. Appl. Phys. 117, 174101 (2015); 10.1063/1.4919705

Effect of Dy-substitution on the structural, vibrational, and multiferroic properties of $\mathrm{BiFeO} 3$ nanoparticles J. Appl. Phys. 115, 214109 (2014); 10.1063/1.4881529

Ferromagnetic, ferroelectric properties, and magneto-dielectric effect of Bi4.25La0.75Fe0.5Co0.5Ti3O15 ceramics

Appl. Phys. Lett. 102, 072904 (2013); 10.1063/1.4793305

Observation of magnetoelectric coupling in $\mathrm{Bi} 0.7 \mathrm{Dy} 0.3 \mathrm{FeO} 3$ thin films at room temperature Appl. Phys. Lett. 93, 132906 (2008); 10.1063/1.2994692

Room temperature multiferroic properties of single-phase ( Bi 0.9 La 0.1 ) Fe O 3 - Ba ( Fe 0.5 Nb 0.5 ) O 3 solid solution ceramics

Appl. Phys. Lett. 90, 042908 (2007); 10.1063/1.2434182

MIT LINCOLN

LABORATORY CAREERS

Discover the satisfaction of innovation and service

to the nation
- Space Control

- Air \& Missile Defense

- Communications Systems \& Cyber Security

- Intelligence, Surveillance and

Reconnaissance Systems

- Advanced
Electronics
- Tactical Systems
- Homeland
Protection
- Air Traffic Control

LINCOLN LABORATORY

MassachusetTS Institute of TeChNOLOGY

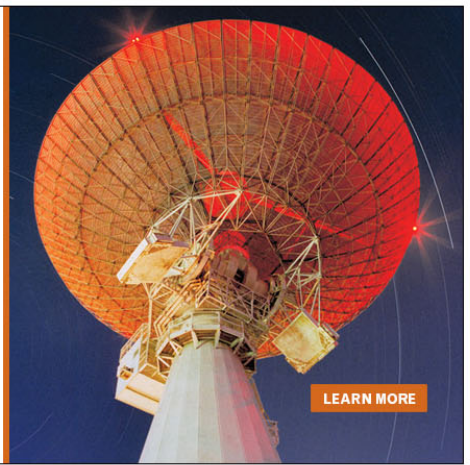




\title{
Observation of room temperature saturated ferroelectric polarization in Dy substituted $\mathrm{BiFeO}_{3}$ ceramics
}

\author{
Shuxia Zhang, ${ }^{1}$ Lei Wang, ${ }^{1}$ Yao Chen, ${ }^{1}$ Dongliang Wang, ${ }^{1}$ Yingbang Yao, ${ }^{2}$ \\ and Yanwei $\mathrm{Ma}^{1, \text { a) }}$ \\ ${ }^{1}$ Institute of Electrical Engineering, Chinese Academy of Sciences, P. O. Box 2703, Beijing 100190, China \\ ${ }^{2}$ Imaging and Characterization Core Lab, King Abdullah University of Science and Technology, \\ Thuwal 23955-6900, Saudi Arabia
}

(Received 22 December 2011; accepted 18 February 2012; published online 6 April 2012)

High quality $\mathrm{Bi}_{1-x} \mathrm{Dy}_{x} \mathrm{FeO}_{3}(0 \leq x \leq 0.15)$ ceramics have been fabricated by sintering Dy-doped $\mathrm{BiFeO}_{3}(\mathrm{BFO})$ precursor powders at a low temperature of $780^{\circ} \mathrm{C}$. The magnetic properties of $\mathrm{BFO}$ were improved by the introduction of $\mathrm{Dy}$ on the Bi-site. More importantly, well saturated ferroelectric hysteresis loops and polarization switching currents have been observed at room temperature. A large remnant polarization $\left(2 P_{\mathrm{r}}\right)$ value of $62 \mu \mathrm{C} / \mathrm{cm}^{2}$ is achieved, which is the highest value reported so far for rare-earth-doped BFO ceramics. Moreover, mechanisms for improved multiferroic properties depending on chemical doping-caused structure evolutions have also been discussed. (C) 2012 American Institute of Physics. [http://dx.doi.org/10.1063/1.3702405]

\section{INTRODUCTION}

Multiferroic materials exhibit multiferroism and magnetoelectricity, pointing to multifunctional applications. Such materials offer extra degrees of freedom in the design of new functional devices, which may either simplify the operation of present device structures or offer new architecture. ${ }^{1,2}$ In recent years, multiferroic materials have been extensively studied for pursuing their possible technical applications, including spintronics and multiple-state memories. ${ }^{3-5}$ Besides, the interest in multiferroics is stimulated by the enthusiasm of exploring the fundamental physics leading to multiferroism and the rich phenomena arising from coupling between magnetic and ferroelectric orderings. ${ }^{5,6}$ However, the naturally existing ferroelectromagnetic materials are rare, due to the mutually exclusive nature of magnetism and the electric polarization phenomenon in most solids. ${ }^{4,7}$ Furthermore, the vast majority of compounds in which ferroelectricity and magnetism are coupled have low ordering temperatures, and a room temperature (RT) operation has not been demonstrated yet. As a special case, $\mathrm{BiFeO}_{3}$ (BFO) is a rhombohedrally distorted perovskite with space group $R 3 \mathrm{c}$ at RT and well known for its high ferroelectric Curie $\left(T_{C} \sim 1103 \mathrm{~K}\right)$ and antiferromagnetic Néel $\left(T_{N} \sim 643 \mathrm{~K}\right)$ temperatures. ${ }^{2,8}$ Incidentally, BFO shows the ferroelectricity arising from $\mathrm{Bi}^{3+}$ lone-pair $6 \mathrm{~S}^{2}$, while not of the magnetic origin. The critical drawbacks of this material, however, is its antiferromagnetic nature and low electrical resistivity. ${ }^{9,10}$ So synthesis of multiferroic materials possessing simultaneously ferroelectric and ferromagnetic properties is still an interesting, but challenging subject.

As proved previously, ${ }^{9}$ BFO has a G-type antiferromagnetic structure, which is cycloidally modulated with a long periodicity of $62 \mathrm{~nm}$. Recent recognition of the possibility to enhance magnetic properties of BFO by inducing lattice

\footnotetext{
a) Author to whom correspondence should be addressed. Electronic mail: ywma@mail.iee.ac.cn. Tel: +86108254 7129. Fax: +86108254 7137.
}

distortion, which may cause collapse of spiral spin structures in BFO, has motivated numerous significant studies. ${ }^{11-15}$ However, the RT ferroelectricity in bulk BFO still raises many questions. Some BFO-based ceramics have been considered to exhibit weak ferromagnetism and ferroelectricity simultaneously, ${ }^{12-17}$ but supporting evidences for ferroelectric properties are mostly indirect characteristics. The most indisputable means to substantiate the ferroelectric properties of a material is to measure its polarization hysteresis loops. Yet to get a clear polarization loop in bulk BFO without any effects of extrinsic parameters is usually difficult, because the synthesized samples are always too conductive. It has been proposed that the leakage behavior in the material is mainly caused by the volatile nature of $\mathrm{Bi}$, which will lead to the formation of oxygen deficiency and $\mathrm{Fe}^{2+} \cdot{ }^{18,19} \mathrm{Up}$ to now, there have been only a few reports on RT-saturated ferroelectric hysteresis loops for BFO-based ceramics. ${ }^{14,15}$ In the present investigation, high quality Dy substituted BFO ceramics have been prepared by sintering the corresponding precursor powders at $780^{\circ} \mathrm{C}$. The sintering temperature is greatly decreased by comparing it with that used in traditional solid state reaction methods (usually beyond $850^{\circ} \mathrm{C}$ [Refs. 11-17]) and even lower than the melting point of $\mathrm{Bi}_{2} \mathrm{O}_{3}$ (which melts at a temperature slightly above $800^{\circ} \mathrm{C}$ [Ref. 20]). By using this low temperature sintering technique, high quality $\mathrm{Bi}_{1-x} \mathrm{Dy}_{x} \mathrm{FeO}_{3}$ ceramics have been achieved with their excellent RT ferroelectric and weak ferromagnetic properties. Moreover, the mechanisms for improved magnetism and electric properties in $\mathrm{Bi}_{1-x} \mathrm{Dy}_{x} \mathrm{FeO}_{3}$ ceramics, depending on chemical doping-caused structure evolutions, have also been investigated.

\section{EXPERIMENTAL PROCEDURES}

The precursor powders of Dy-substituted BFO were prepared by a microwave-assisted sol-gel self-combustion method using analytical reagent-grade bismuth nitrate $\left[\mathrm{Bi}\left(\mathrm{NO}_{3}\right)_{3} \cdot 5 \mathrm{H}_{2} \mathrm{O}\right]$, ferric nitrate $\left[\mathrm{Fe}\left(\mathrm{NO}_{3}\right)_{3} \cdot 9 \mathrm{H}_{2} \mathrm{O}\right]$, and high-purity $\mathrm{Dy}_{2} \mathrm{O}_{3}$ as starting materials. Firstly, a 
stoichiometric amount of $\mathrm{Dy}_{2} \mathrm{O}_{3}$ was dissolved in certain diluted nitric acid under constant stirring. Next, bismuth nitrate together with ferric nitrate in a proper mole ratio was also dissolved in the above solutions. Then, glycine was added as fuel. Subsequently, the resulting homogeneous solution was put into a $1000-\mathrm{W}$ microwave oven for autoignition experiments, resulting in the formation of voluminous powders. Afterwards, the obtained precursor powders were pressed into disk samples $(11 \mathrm{~mm}$ diameter, $\sim 1 \mathrm{~mm}$ thickness) after grounding in an agate mortar for a moment. Finally, the pressed samples were annealed at $780^{\circ} \mathrm{C}$ for $2 \mathrm{~h}$ in air. The sintering will result in compact ceramics, with an average density value of $\sim 7.6 \mathrm{~g} / \mathrm{cm}^{3}$, which is about $91 \%$ of the theoretical value of $8.33 \mathrm{~g} / \mathrm{cm}^{3}$. It is worth noting here that the annealing temperature is much lower than that in a traditional solid state reaction method (usually higher than $820^{\circ} \mathrm{C}$ ). Here, we denoted the samples as S1, S2, S3, and S4 according to composition $\mathrm{Bi}_{1-x} \mathrm{Dy}_{x} \mathrm{FeO}_{3}$, with $x=0,0.05$, 0.1 , and 0.15 , respectively. The crystal structure identification was examined by an X-ray diffraction (XRD) machine with $\mathrm{Cu} \mathrm{K} \alpha$ radiation. The magnetic measurement was performed on a vibrating sample magnetometer in a physical properties measurement system (PPMS). Polarizations, displacement currents, and leakage measurements were investigated at RT using a ferroelectric test system (TF Analyzer 2000, aixACCT). Before the ferroelectric and leakage behavior measurements, the sintered disk samples were polished down to 0.2-mm thickness and then sputtered with Pt electrodes for the corresponding studies.

\section{RESULTS AND DISCUSSIONS}

Figure 1(a) shows the XRD patterns for all the samples. It can be seen that the reflection peaks in the traces of pure compound (S1) can be successfully indexed as a rhombohedral distortion perovskite structure with a space group of $R 3 c$. In the case of doped samples, the spectra collected for $\mathrm{S} 2$ and $\mathrm{S} 3$ still can be indexed using the rhombohedral cell. However, the XRD pattern of S4 mainly shows an orthorhombic $\mathrm{DyFeO}_{3}$-like profile (space group Pnma). ${ }^{11,12}$ Note that no trace of other impurity phases can be detected for all ceramics. From Fig. 1(a), it is clear that, with increasing Dy content up to 0.1 , the relative diffraction intensity of the (104) peak decreased, whereas that of the (110) one increased. Meanwhile, some diffraction peaks coming from rhombohedral $R 3 \mathrm{c}$ structure, such as the (006) diffraction peak, become weak with the increase of $x$ and tend to disappear near $x=0.1$. The magnified patterns in the vicinity of $2 \theta=32^{\circ}$ are shown in Fig. 1(b), which shows that the (104) and (110) reflections are clearly separated in pure sample (S1), but the reflections shift to large angles and partially overlap with increasing Dy content up to 0.1. The large angle shift indicates that the unit cells in these samples are shrinking, which may be explained by the fact that the ionic radius of $\mathrm{Dy}^{3+}$ is smaller than that of $\mathrm{Bi}^{3+} \cdot{ }^{21}$ Further increase in Dy content to 0.15 gives an obvious structure transformation from rhombohedral to orthorhombic. The XRD results confirm that the substitution of Dy into the Bi

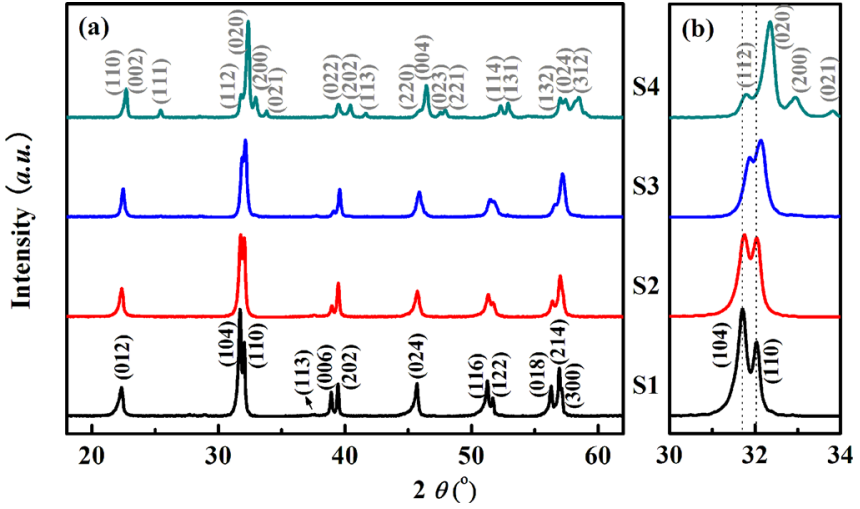

FIG. 1. (a) $\mathrm{X}$ ray diffraction patterns of $\mathrm{Bi}_{1-x} \mathrm{Dy}_{x} \mathrm{FeO}_{3}(0 \leq x \leq 0.15)$ ceramics and (b) magnified patterns in the vicinity of $2 \theta=32^{\circ}$.

site in BFO will lead to changes in lattice parameter and crystal structure.

The spin cycloid of BFO is correlated to its $R 3 \mathrm{c}$ structure. Therefore, it is deduced that the lattice distortions might destruct the inhomogeneous spin structure. To study the magnetic behaviors in these samples, Fig. 2 panels (a)-(d) plot the RT magnetization hysteresis $(M-H)$ loops of S1, S2, S3, and S4, respectively. The strikingly enhanced magnetic properties by Dy substitution are observed in the figure, similar to the magnetization evolutions caused by other rare-earth ion A-site doping. ${ }^{13-16}$ In contrast to the typical antiferromagnetic S1, with an almost linear magnetic field dependence of the magnetization, S2 exhibits a weak ferromagnetism, with a small remnant magnetization $\left(M_{\mathrm{r}}\right)$ value of $0.021 \mathrm{emu} / \mathrm{g}$. With further increasing substitution content, the corresponding $M_{\mathrm{r}}$ values are 0.213 and $0.313 \mathrm{emu} / \mathrm{g}$ for S3 and S4, respectively. In addition to the improved remnant magnetization by Dy substitution, the $M_{\max }$ (magnetization at $7 \mathrm{~T}$ ) value is also increased. The magnetization enhancement in Dy-substituted samples can be attributed to the collapse of the space-modulated spin structure. As illustrated in Fig. 1, the changes in lattice parameters and crystal structure give evidence for continuing collapse of the spiral spin structure.

The polarization versus applied electrical field $(P-E)$ loops for each sample under various driving electric field with the fixed frequency of $100 \mathrm{~Hz}$ are depicted in Figs. 3(a)-3(d), respectively, while the corresponding polarization current dependence on the electric field is also plotted along with each $P-E$ loop using dot dashed lines in Figs. 3(a)-3(d). The double remnant polarization $\left(2 P_{\mathrm{r}}\right)$ value of $21 \mu \mathrm{C} / \mathrm{cm}^{2}$ is obtained for $\mathrm{S} 1$ at a maximum applied electric field of $160 \mathrm{kV} / \mathrm{cm}$. However, note that freely movable charges appear to contribute significantly to the electrical hysteresis loops, as manifested by the rounded features of the loops, which means that the electrical leakage is still severe in S1. In contrast, the room temperature $P-E$ response shows well square $P-E$ loops for S2 and S3 ceramics, and the samples are not subjected to dielectric breakdown, even at the maximum applied electric field of $200 \mathrm{kV} / \mathrm{cm}$. With this maximum driving field, the measured $2 P_{\mathrm{r}}$ values are 62 and $43 \mu \mathrm{C} / \mathrm{cm}^{2}$, while the coercive fields are 120 and $130 \mathrm{kV} \mathrm{cm}^{-1}$ for $\mathrm{S} 2$ and $\mathrm{S} 3$ ceramics, 

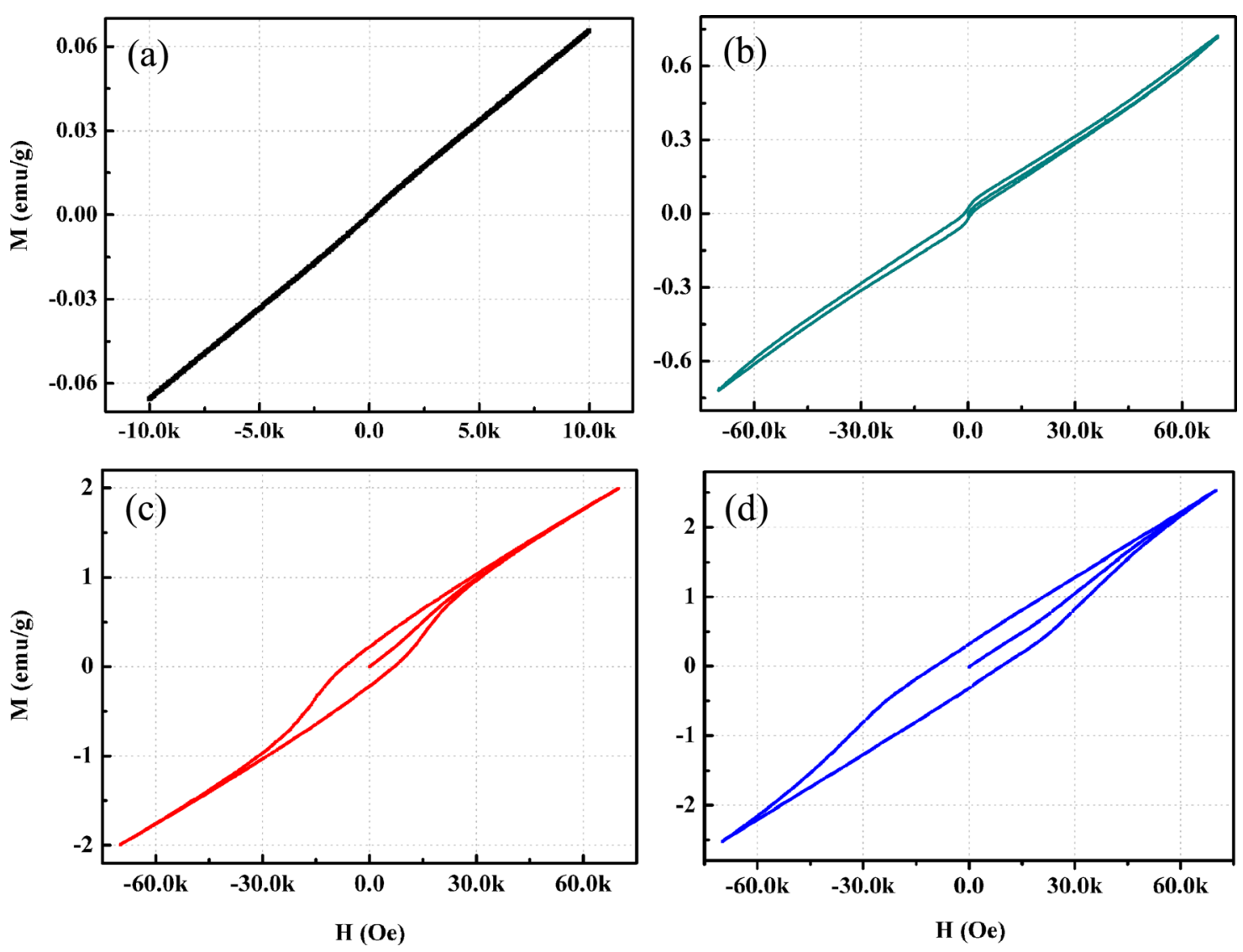

FIG. 2. Magnetization hysteresis $(M-H)$ loops of $\mathrm{Bi}_{1-x} \mathrm{Dy}_{x} \mathrm{FeO}_{3}(0 \leq x \leq 0.15)$ ceramics: (a) $x=0$; (b) $x=0.05$; (c) $x=0.1$; and (d) $x=0.15$.

respectively. Importantly, the $2 P_{\mathrm{r}}$ values obtained in $\mathrm{S} 2$ and S3 ceramics are significantly higher than the highest values reported so far for rare-earth-doped BFO bulk ceramics. ${ }^{14,15,22}$ However, it seems difficult to induce polarization reversal if the substitution content is too high, and the $P-E$ loops of $\mathrm{S} 4$ are far from saturation with the maximum applied field of $200 \mathrm{kV} / \mathrm{cm}$.

As the applied electric field is ramped to the coercive electric fields for both S2 and S3, the displacement currents depicted with dot dashed lines in Figs. 3(b) and 3(c) exhibit a sharp increase, demonstrating the occurrence of ferroelectric switching in these ceramics. Furthermore, the ferroelectric switching-induced abrupt current peaks are rather symmetric with respect to the voltage polarity. Yet, even displacement current shows an increase trend in S1; the current peaks are not observed probably due to the low electric breakdown field and the large leakage current of pure BFO. The low electric breakdown field may restrict the switching of ferroelectric domains, and the large leakage currents will overlay the domain switching current. Similarly, the current peaks and even dielectric breakdown do not occur in $\mathrm{S} 4$ with the maximum driving electric field of $200 \mathrm{kV} / \mathrm{cm}$ either, probably revealing the poor ferroelectric properties of S4 ceramics. It is well known that the polarization within the domains can be switched by an applied external electric field. Polarization switching in BFO is especially important, because it might also switch the antiferromagnetic/ferromagnetic orientation, which is necessary for magnetoelectric coupling. ${ }^{23}$ These observed saturated $P-E$ loops and successful ferroelectric switching in weak ferromagnetic $\mathrm{Bi}_{1-x} \mathrm{Dy}_{x} \mathrm{FeO}_{3}$ ceramics may imply that decent $\mathrm{BFO}$ ferroelectromagnetic materials can be achieved by moderate Dy substitution.

Electrical properties of the ceramics were also determined. Figure 4 describes the electric field dependence of leakage current density $(J-E)$ at RT. Under the same test electric field, the Dy-substituted BFO has a smaller leakage current density than the pure BFO, and the smallest leakage current density is obtained in the sample with $10 \%$ Dy. When the test electric field equals to $20 \mathrm{kV} / \mathrm{cm}$, for example, the leakage current densities of S3 is $5.2 \times 10^{-6} \mathrm{~A} / \mathrm{cm}^{2}$ and the value of resistivity is evaluated to be $6.62 \times 10^{9} \Omega \mathrm{cm}$, suggesting highly insulating characteristics of the sample at RT.

To measure the saturation polarization for ferroelectric materials, high quality samples with low conductivity are required to suffer high electric fields. A notorious problem in BFO is the volatilization of $\mathrm{Bi}$, which will lead to the deviation from oxygen stoichiometry, thus creating oxygen vacancies and inducing valence fluctuation of $\mathrm{Fe}(+3$ to +2 state) ions, ${ }^{18,19}$ resulting in high conductivity. The low sintering temperature of $780{ }^{\circ} \mathrm{C}$ is beneficial to avoid this stoichiometry deviation, but not sufficient to get high quality ceramics, because that pure BFO (S1) is still very leaky and shows antiferromagnetic character. With the fabrication technique developed in this work, Dy substitution can further decrease leakage current density and simultaneously suppress the antiferromagnetic spin structure in BFO. As a result, well-saturated ferroelectric hysteresis loops and 

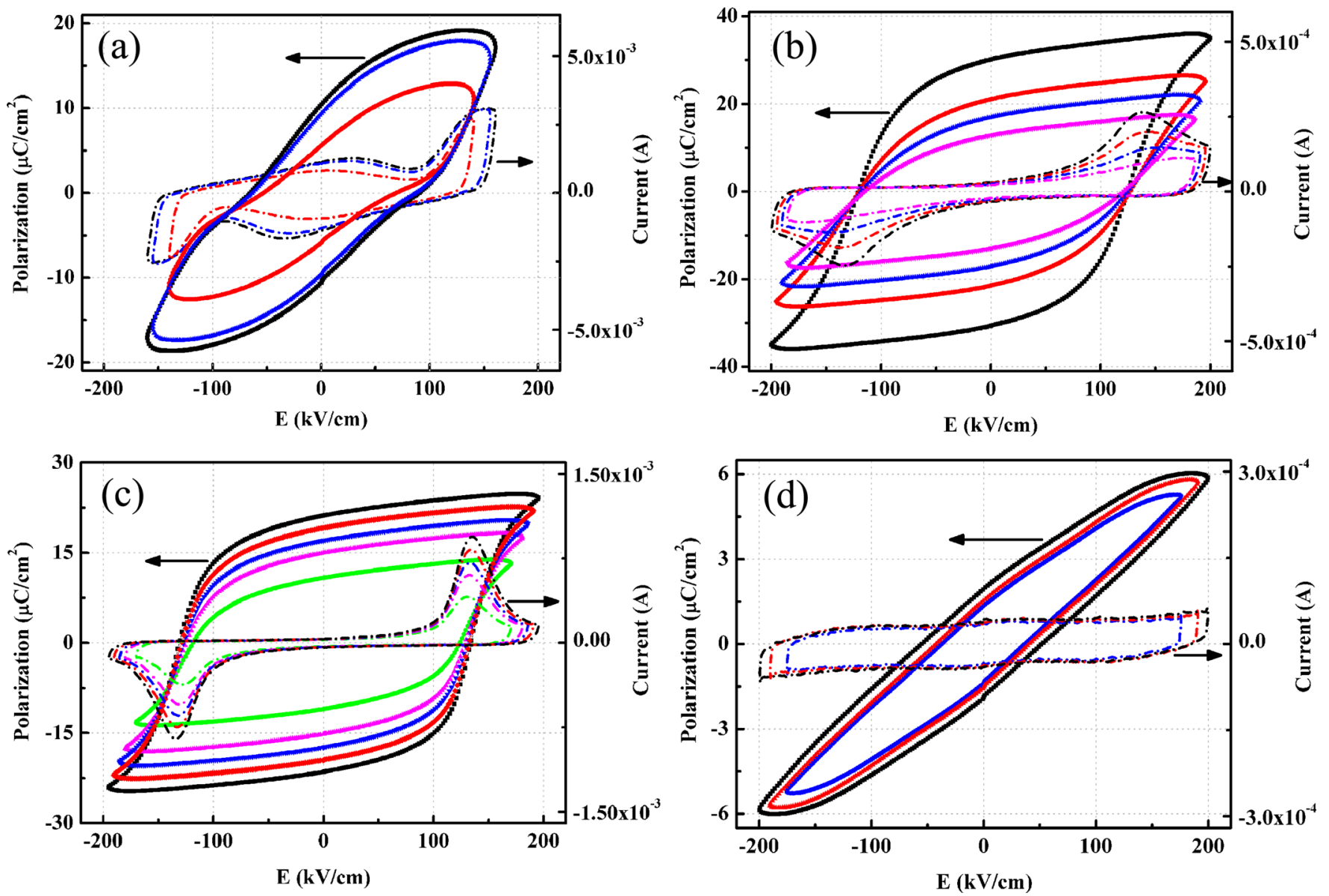

FIG. 3. Ferroelectric polarization and displacement current vs electric field as measured for $\mathrm{Bi}_{1-x} \mathrm{Dy}_{x} \mathrm{FeO}_{3}(0 \leq x \leq 0.15)$ ceramics: (a) $x=0$; (b) $x=0.05$; (c) $x=0.1$; and (d) $x=0.15$.

obviously improved magnetic properties are observed in our $\mathrm{Bi}_{1-x} \mathrm{Dy}_{x} \mathrm{FeO}_{3}$ ceramics.

Due to the exact measurements of magnetization and saturated polarization hysteresis loops, mechanisms for improved multiferroic properties depending on chemical doping-caused structure evolutions have been discussed. In the $\mathrm{Bi}_{1-x} \mathrm{Dy}_{x} \mathrm{FeO}_{3}$ system, it is clear that low concentration substitution only induces lattice parameter changes, while

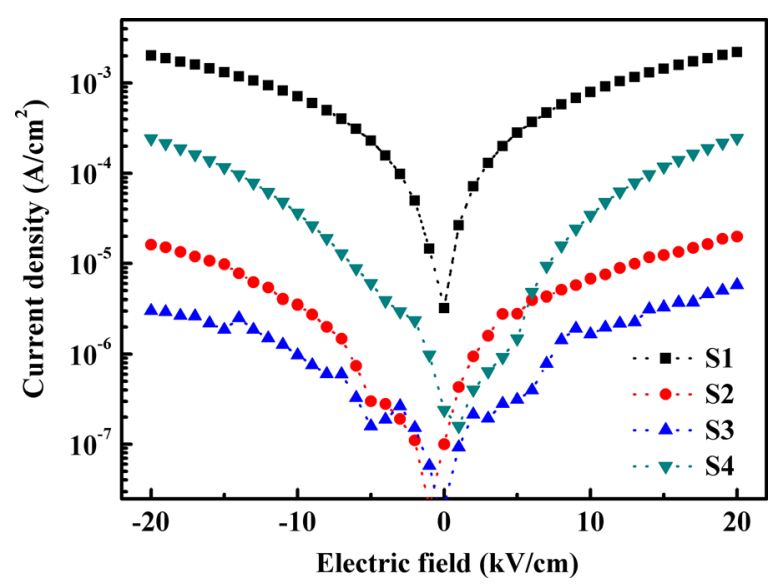

FIG. 4. Leakage current as a function of applied electrical field in $\mathrm{Bi}_{1-x} \mathrm{Dy}_{x} \mathrm{FeO}_{3}$ $(0 \leq x \leq 0.15)$ ceramics with $x=0$ (S1), 0.05 (S2), 0.1 (S3), and 0.15 (S4), respectively. high concentration substitution will finally give rise to a structure transition from rhombohedral to orthorhombic. The lattice distortions result in collapse of spiral spin structures in BFO, which is responsible for the greatly increased magnetization in the substituted ceramics. In addition, Dy substitution is beneficial for decreasing the leakage behavior in the material. Low leakage current density, well-saturated polarization loops, and excellent ferroelectric switching are observed in the 5\% and 10\% Dy-substituted BFO ceramics, suggesting that the magnetic and ferroelectric properties of BFO can be simultaneously improved through Dy substitution. Yet too much substitution will degrade the ferroelectricity of the material, as evidenced in S4; the material displays no ferroelectric switching. We infer that this results from two main reasons: Firstly, a dominant orthorhombic structure occurs for S4 with 15\% Dy substitution. The orthorhombic structure is more centrosymmetric, which, in principle, does not allow ferroelectricity. ${ }^{21,24}$ Therefore, it is much more difficult to induce polarization in $\mathrm{S} 4$, and a smaller electrical polarization is expected, even under an ultrahigh poling electric field. Secondly, it is well known that the A-site $\mathrm{Bi}$ ions via the $6 \mathrm{~S}^{2}$ lone-pair mechanism leads to the ferroelectricity in $\mathrm{BFO}$, so the substitution of $\mathrm{Bi}^{3+}$ with $\mathrm{Dy}^{3+}$ will weaken the stereochemical activity of the lonepair, due to the spherical distribution of electron density in rare-earth $\mathrm{Dy}^{3+}$ ions. ${ }^{24}$ This feature is potentially consistent 
with the hypothesis proposed by Catalan and Scott; ${ }^{25}$ the substitution of $\mathrm{Dy}^{3+}$ can be regarded as turning off the lonepair activity of $\mathrm{Bi}^{3+}$ (i.e., turning off the ferroelectricity), which will ultimately degrade the spontaneous polarization in the material.

\section{CONCLUSION}

In summary, BFO ceramic samples with different amounts of Dy substitution have been synthesized and characterized. It is found that the substitution of Dy causes noticeable lattice distortions of the ceramics. Besides the obviously improved magnetic properties, we show here that Dy-substituted BFO has high enough resistivity for us to measure the clear polarization loops at RT. The coexistence of ferromagnetism and ferroelectricity has been confirmed in the BFO ceramics with $5 \%$ and $10 \%$ Dy, which suggests that doping of rare earth elements is important, not only for magnetic improvement, but also for ferroelectric enhancement, and namely important for overall multiferroic improvement.

\section{ACKNOWLEDGMENTS}

This work was partially supported by the National Natural Science Foundation of China (Grant No. 51025726).

\footnotetext{
${ }^{1}$ M. Fiebig, T. Lottermoser, D. Fröhlich, A. V. Goltsev, and R. V. Pisarev, Nature 419, 818 (2002).

${ }^{2}$ J. Wang, J. B. Neaton, H. Zheng, V. Nagarajan, S. B. Ogale, B. Liu, D. Viehland, V. Vaithyanathan, D. G. Schlom, U. V. Waghmare, N. A. Spaldin, K. M. Rabe, M. Wutting, and R. Ramesh, Science 299, 1719 (2003).

${ }^{3}$ H. Bea, M. Gajek, M. Bibes, and A. Barthelemy, J. Phys.: Condens. Matter 20, 434231 (2008).

${ }^{4}$ N. A. Hill, J. Phys. Chem. B 104, 6694 (2000).
}

${ }^{5}$ R. Ramesh and N. A. Spaldin, Nature Mater. 6, 21 (2007).

${ }^{6}$ M. Fiebig, J. Phys. D: Appl. Phys. 38, R123 (2005).

${ }^{7}$ D. I. Khomskii, J. Magn. Magn. Mater. 306, 1 (2006).

${ }^{8}$ F. Kubel and H. Schmid, Acta Crystallogr., Sect. B: Struct. Sci. 46, 698 (1990).

${ }^{9}$ I. Sosnowska, T. Peterlin-Neumaier, and E. Steichele, J. Phys. C 15, 4835 (1982).

${ }^{10}$ J. R. Teague, R. Gerson, and W. J. James, Solid State Commun. 8, 1073 (1970).

${ }^{11}$ S. Zhang, W. Luo, D. Wang, and Y. Ma, Mater. Lett. 63, 1820 (2009).

${ }^{12}$ V. A. Khomchenko, D. V. Karpinsky, A. L. Kholkin, N. A. Sobolev, G. N. Kakazei, J. P. Araujo, I. O. Troyanchuk, B. F. O. Costa, and J. A. Paixão, J. Appl. Phys. 108, 074109 (2010).

${ }^{13}$ G. L. Yuan, S. W. Or, J. M. Liu, and Z. G. Liu, Appl. Phys. Lett. 89, 052905 (2006).

${ }^{14}$ G. L. Yuan and S. W. Or, J. Appl. Phys. 100, 024109 (2006).

${ }^{15}$ S. T. Zhang, L. H. Pang, Y. Zhang, M. H. Lu, and Y. F. Chen, J. Appl. Phys. 100, 114108 (2006).

${ }^{16}$ V. A. Khomchenko, D. A. Kiselev, I. K. Bdikin, V. V. Shvartsman, P. Borisov, W. Kleemann, J. M. Vieira, and A. L. Kholkin, Appl. Phys. Lett. 93, 262905 (2008).

${ }^{17}$ Z. Yan, K. F. Wang, J. F. Qu, Y. Wang, Z. T. Song, and S. L. Feng, Appl. Phys. Lett. 91, 082906 (2007).

${ }^{18}$ Z. Hu, M. Li, B. Yu, L. Pei, J. Liu, J. Wang, and X. Zhao, J. Phys. D: Appl. Phys. 42, 185010 (2009).

${ }^{19}$ Y. P. Wang, L. Zhou, M. F. Zhang, X. Y. Chen, J. M. Liu, and Z. G. Liu, Appl. Phys. Lett. 84, 1731 (2004).

${ }^{20}$ R. Palai, R. S. Katiyar, H. Schmid, P. Tissot, S. J. Clark, J. Robertson, S. A. T. Redfern, G. Catalan, and J. F. Scott, Phys. Rev. B 77, 014110 (2008).

${ }^{21}$ W. M. Zhu, L. W. Su, Z. G. Ye, and W. Ren, Appl. Phys. Lett. 94, 142908 (2009).

${ }^{22}$ S. Y. Wang, X. Qiu, J. Gao, Y. Feng, W. N. Su, J. X. Zheng, D. S. Yu, and D. J. Li, Appl. Phys. Lett. 98, 152902 (2011).

${ }^{23}$ Y. H. Chu, L. W. Martin, M. B. Holcomb, M. Gajek, S.-J. Han, Q. He, N. Balke, C.-H. Yang, D. Lee, W. Hu, Q. Zhan, P.-L. Yang, A. Fraile-Rodriguez, A. Scholl, S. X. Wang, and R. Ramesh, Nature Mater. 7, 478 (2008).

${ }^{24}$ I. O. Troyanchuk, M. V. Bushinsky, D. V. Karpinsky, O. S. Mantytskaya, V. V. Fedotova, and O. I. Prochnenko, Phys. Status Solidi B 246, 1901 (2009).

${ }^{25}$ G. Catalan and J. F. Scott, Adv. Mater. 21, 2463 (2009). 